\title{
Formation of relaxed SiGe on the buffer consists of modified SiGe stacked layers by Si pre-intermixing
}

\author{
P.S. Chen ${ }^{\mathrm{a}, *}$, S.W. Lee ${ }^{\mathrm{b}}$, M.H. Lee ${ }^{\mathrm{c}}$, C.W. Liu ${ }^{\mathrm{d}}$ \\ a Department of Materials Science and Engineering, MingShin University of Science and Technology, No. 1 Hsin-Hsin Road, Hsin-Feng, Hsin Chu, 30401, Taiwan, ROC \\ ${ }^{\mathrm{b}}$ Institute of Materials Science and Engineering, National Central University, Chungli 32001, Taiwan, ROC \\ ${ }^{\mathrm{c}}$ Institute of Electro-optical Science and Technology, National Taiwan Normal University, Taipei, Taiwan, ROC \\ ${ }^{\mathrm{d}}$ Department of Electrical Engineering, National Taiwan University, Taipei, Taiwan, ROC
}

\section{A R T I C L E I N F O}

\section{Article history:}

Available online 15 March 2008

\section{PACS:}

68.65. Hb

61.66.Dk

61.72.Lk

68.37.Lp

\section{Keywords:}

Relaxed SiGe

UHVCVD

SiGe islands

Strained Si

\begin{abstract}
A B S T R A C T
High-quality relaxed SiGe films on Si $\left(\begin{array}{llll}0 & 0 & 1\end{array}\right)$ have been demonstrated with a buffer layer containing modified SiGe ( $\mathrm{m}$-SiGe) islands in ultra-high vacuum chemical vapor deposition (UHV/CVD) system. The $\mathrm{m}$-SiGe islands are smoothened by capping an appropriate amount of Si and the subsequent annealing for $10 \mathrm{~min}$. This process leads to the formation of a smooth buffer layer with non-uniform Ge content. With the $\mathrm{m}$-SiGe-dot multilayer as a buffer layer, the 500 -nm-thick uniform $\mathrm{Si}_{0.8} \mathrm{Ge}_{0.2}$ layers were then grown. These $\mathrm{m}$-SiGe islands can serve as effective nucleation centers for misfit dislocations to relax the SiGe overlayer. Surface roughness, strain relaxation, and crystalline quality of the relaxed SiGe overlayer were found to be a function of period's number of the m-SiGe-dot multilayer. By optimizing period number in the buffer, the relaxed $\mathrm{Si}_{0.8} \mathrm{Ge}_{0.2}$ film on the 10 -period $\mathrm{m}$-SiGe-dot multilayer was demonstrated to have a threading dislocation density of $2.0 \times 10^{5} \mathrm{~cm}^{-2}$ and a strain relaxation of $89 \%$.
\end{abstract}

(c) 2008 Elsevier B.V. All rights reserved.

\section{Introduction}

The growth of self-assembled Ge islands on $\mathrm{Si}$ has been intensively investigated for the promising applications of future electronic and optoelectronic devices in recent years [1-3]. Deposition of Ge on (l0 01 ) Si results in the formation of a 3-4 ML wetting layer. Beyond this critical thickness, the threedimensional islands are known to develop by the StranskiKrastanow (S-K) growth mode [1]. Earlier studies reported some morphological evolution and structural changes of the Ge islands occurred during the process of capping with Si [4-6]. Owing to the lack of surface flatness, it may be detrimental to the integrity of the following epitaxial Si or SiGe layer. Therefore, it remains an important issue to study the evolution of surface morphology of Ge islands during the Si cap layer, which depends on the growth temperature, the deposition amount, and the deposition rate of Si. Dental et al. have presented that surface smoothing of Ge islands can be achieved by introducing a Si cap layer [7]. In the previous investigation of evolution of Ge islands grown at $600{ }^{\circ} \mathrm{C}$ during the initial stages of $\mathrm{Si}$ encapsulation, drastic changes of shape and

\footnotetext{
* Corresponding author. Tel.: +886 3 5593142; fax: +886 36007558 .

E-mail address: pschen@must.edu.tw (P.S. Chen).
}

surface morphology of the SiGe films have been observed [4]. It has also been demonstrated that buried Ge islands can relieve the strain between SiGe overlay and Si substrate in the sandwich structure of SiGe/Ge/Si [8]. To grow high quality and strain-relaxed SiGe epilayers, several methods, such as use of different buffers including a low-temperature $\mathrm{Si}$, an ion-implanted $\mathrm{Si}$ or compliant substrates, have been employed [9-12]. In the previous study, the high-quality relaxed SiGe films using a buffer containing Ge islands have been achieved. Effective electron mobility for the strained $\mathrm{Si}$ device was found $90 \%$ higher than that of the Si control device [13]. In this work, we demonstrated this method for fabricating a relaxed SiGe films with a buffer layer containing $\mathrm{m}$-SiGe dots, which were treated with the alloying of encapsulated $\mathrm{Si}$ on $\mathrm{Ge}$ islands and an in situ annealing process. This process results in smoothing of the growth surface with a non-uniformity Ge concentration, which serves as the temple for the following SiGe overlayers. The effects of periods of m-SiGe islands on the relaxation, surface morphology and crystallinity of the resulting SiGe epilayers will be discussed.

\section{Experiment}

Ten to $25 \Omega \mathrm{cm}, 4$ in. diameter p-type ( $\left.\begin{array}{lll}0 & 0 & 1\end{array}\right)$-oriented Si wafers were used in the present study. All structures investigated in this 
work were grown at 550 or $600{ }^{\circ} \mathrm{C}$ in a commercially available multi-wafer UHV/CVD system. $\mathrm{SiH}_{4}$, and $5 \% \mathrm{GeH}_{4}$ diluted in $\mathrm{He}$ were used as a $\mathrm{Si}$, and Ge source. A 60-nm-thick Si buffer layer was first grown on the wafer surface. Then a Ge layer of 13.1 eq-MLs was deposited to form a high uniformly self-assembled islands under S-K growth mode [14]. The amount of encapsulated Si layer above the Ge films were chosen to be 14, 21 and 28 eq-MLs. As the deposition was finished, the wafers were kept in the deposition
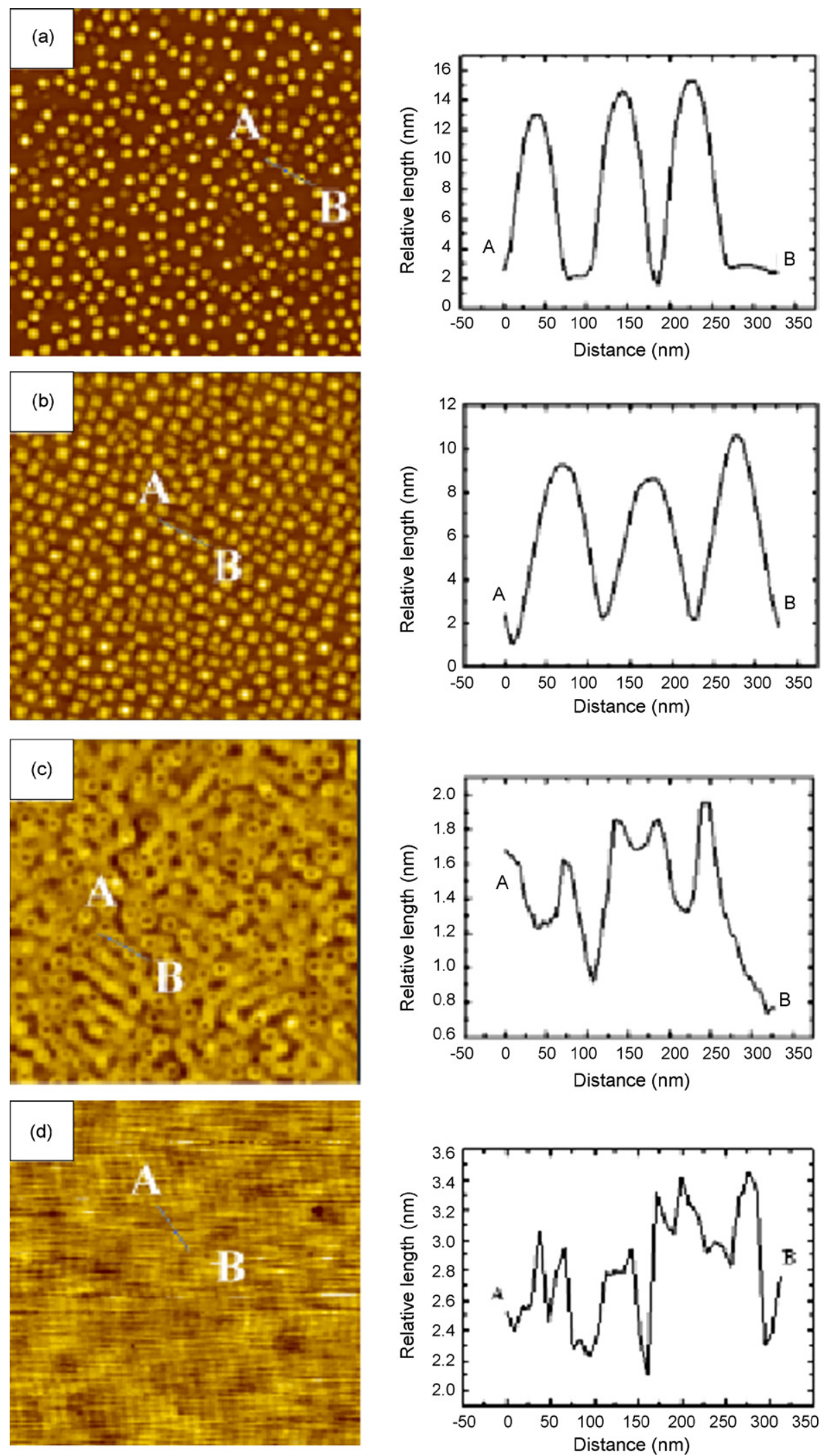

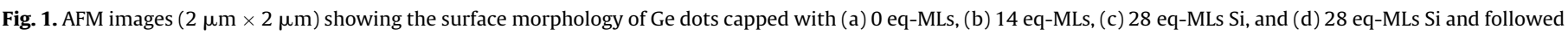
by an in situ annealing for $10 \mathrm{~min}$. The right figures are the corresponding line profile. 
Table 1

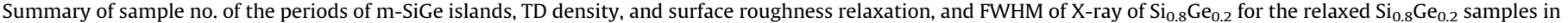
this works

\begin{tabular}{|c|c|c|c|c|c|}
\hline Sample no. & Periods of m-SiGe islands & TD density $\left(\mathrm{cm}^{-2}\right)$ & Roughness (nm) & Relaxation (\%) & FWHM of X-ray (arcsec) \\
\hline A & 0 & $>10^{9}$ & 12.8 & 51 & 529.7 \\
\hline B & 1 & $>10^{9}$ & 5.2 & 61 & 505.7 \\
\hline C & 5 & $4.4 \times 10^{6}$ & 4.6 & 80 & 485.9 \\
\hline $\mathrm{D}$ & 10 & $2.1 \times 10^{5}$ & 3.1 & 89 & 448.7 \\
\hline $\mathrm{E}$ & 20 & $3.0 \times 10^{6}$ & 4.4 & 85 & 468.3 \\
\hline $\mathrm{F}$ & $g$ & $5.1 \times 10^{5}$ & 6.4 & 88 & 486.7 \\
\hline
\end{tabular}

Here, $g$ indicates the structure of the sample is an 800 -nm-thick $\mathrm{Si}_{0.8} \mathrm{Ge}_{0.2}$ uniform layer with CGB.

chamber for 3 or 10 min, unless otherwise specified. The Ge QDs sample with a 28 eq-MLs Si cap layer, then followed by a $10-\mathrm{min}$ growth interruption was found to be very smooth. We referred these modified Ge dots as m-SiGe islands. Finally, a 500-nm-thick $\mathrm{Si}_{0.8} \mathrm{Ge}_{0.2}$ films were grown on the $1,5,10$, and 20 -period of $\mathrm{m}$-SiGe QDs/Si bilayers with $\mathrm{Si}$ spacer of $20 \mathrm{~nm}$ (samples B-E). For comparison, $500-n m$-thick $\mathrm{Si}_{0.8} \mathrm{Ge}_{0.2}$ films grown on $\mathrm{Si}$ (sample A) and 800 -nm-thick $\mathrm{Si}_{0.8} \mathrm{Ge}_{0.2}$ films grown on the 2 - $\mu$ m-thick conventional graded buffer (CGBs, sample $\mathrm{F}$ ) were also prepared. Experimental data for the relaxed SiGe samples in this work was summarized in Table 1.

The shape and size distribution of Ge islands or m-SiGe dots were characterized by atomic force microscopy (AFM) in tapping mode. Cross-section transmission electron microscopy (XTEM) images revealed more detailed information about size, shape distribution and facets of the $\mathrm{Si} / \mathrm{Ge}$ nanostructures. Energy dispersion spectrometer (EDS) coupled with TEM was utilized to determine the composition of Ge nanostructures. The dislocations of SiGe on $\mathrm{m}$-SiGe islands buffers were investigated by XTEM, and the threading dislocation (TD) density of relaxed SiGe films was further determined by Schimmel etch pit method [15]. The strain relaxation and crystalline quality of SiGe films were characterized by high-resolution X-ray diffraction (HRXRD).

\section{Results and discussion}

As shown in Fig. 1, the AFM images give the evolution of surface topography for Ge islands grown at $600{ }^{\circ} \mathrm{C}$ during the $\mathrm{Si}$ overgrowth. At this growth temperature, a high uniformity of dome-shaped $\mathrm{Ge}$ islands has been achieved. For an uncapped sample, Fig. 1(a) shows the well-known bimodal distribution of $\mathrm{Ge}$ islands. After deposition of 14 eq-MLs Si on the prior Ge islands, the pyramids with $\left\{\begin{array}{lll}1 & 0 & 5\end{array}\right\}$ facets are dominant, and the dome-shaped mounds are absent, as shown in Fig. 1(b). This phenomenon can be explained in terms of the dependence of equilibrium shape of islands on their volume and composition as a result of alloying between $\mathrm{Si}$ and $\mathrm{Ge}$. Our results are consistent with those of the earlier studies for the Si overgrowth on the Ge islands [4,6]. Fig. 1(c) shows the surface morphology of Ge islands with a Si layer of 28 eq-MLs. At this stage, the volcanoshaped islands formed. Fig. 2(a) and (b) shows the XTEM images of uncapped domes and volcano-shaped islands, respectively. It is observed that $\mathrm{Ge}$ islands reduced its heights with the increase of $\mathrm{Si}$ coverage. It is likely that due to the partial relaxation of $\mathrm{Ge}$ dot at the apex, a strain modulation was applied on the encapsulated Si layer. Above the Ge-rich dot, the in-plane lattice of Si-rich capping layer was laterally elongated and exhibited tensile strain. To reduce the accumulated strain energy, the Si atoms migrated from the severely strained regions to the relatively strain-free regions around the Ge-rich QDs and developed the volcano-shaped nanostructure on the surface. The EDS measurement for the volcano-ring shown in Fig. 2(b) shows that the Ge compositions at the core and ridges are about
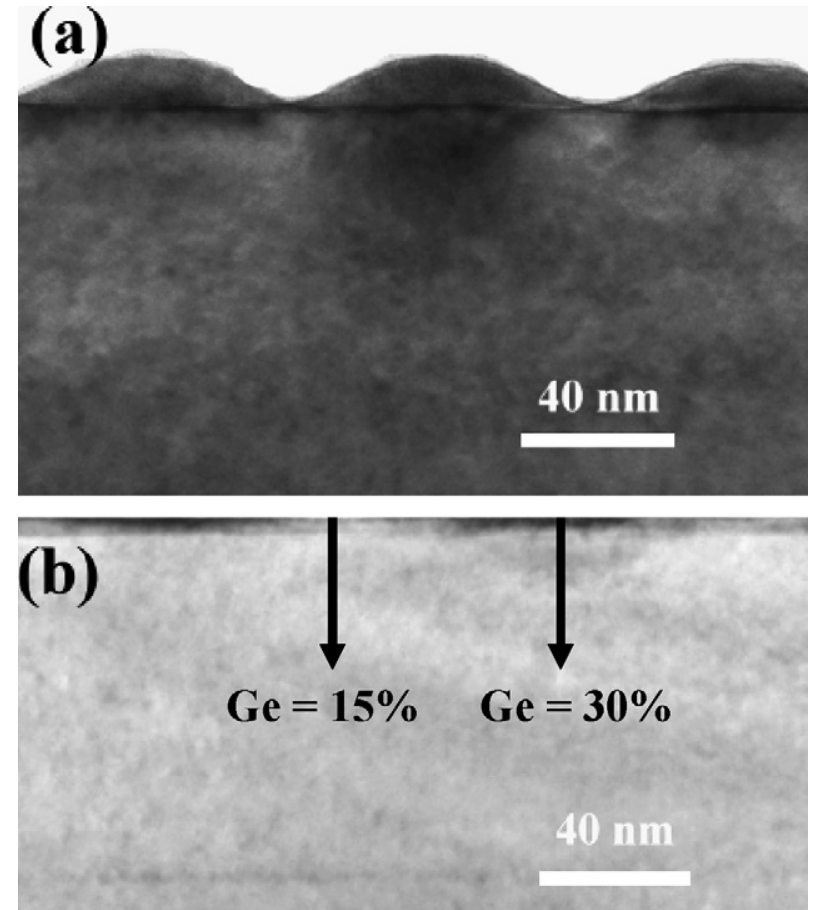

Fig. 2. XTEM image of 13.1 eq-MLs Ge islands capped (a) without and (b) with 28 eq-MLs Si. The Ge content in SiGe was determined by EDS.

30 and 15\%. The results also indicated that substantial intermixing of $\mathrm{Si}$ and Ge occurred during the disappearance of the volcano-shaped islands, which were in situ annealed at $600{ }^{\circ} \mathrm{C}$ for $10 \mathrm{~min}$. With proper growth temperature and enough time, the surface of Ge islands layer could be smoothened with the Si pre-intermixing. As shown in Fig. 3(a), the formation of mounds on the surface of Si/Ge islands with a 20-nm-thick Si cap layer was seen from the observation of AFM. As a result, more stress centers form the top of protruded parts of the corrugated surface and induced a high TD density in the overgrown SiGe epilayers. Therefore, a growth interruption for $10 \mathrm{~min}$ between the end of the alloying of Ge islands with a 28-eq-ML-thick Si and the start of the overgrown Si spacer was necessary to avoid the corrugated surface of the Si/Ge islands. The smooth surface morphology of a $\mathrm{m}$-SiGe/Si layer (RMS roughness $\sim 0.3 \mathrm{~nm}$ ) is shown in Fig. 3(b). Moreover, the surface of 10-period Si/m-SiGe bilayers (Fig. 3(c)) has the RMS roughness of $0.74 \mathrm{~nm}$, which is lower than that of the Ge QDs capped with a 20 -nm-thick Si $(0.95 \mathrm{~nm})$. This result suggested that $\mathrm{m}$-SiGe islands are beneficial in lowering the surface roughness of buffer layer.

As shown in Fig. 4(a), the microstructure of the 500-nm-thick $\mathrm{Si}_{0.8} \mathrm{Ge}_{0.2}$ film grown on $\mathrm{Si}$ was found to have a lot of pile-up dislocations at the interface, which induced by the modified Frank Read (MFR) mechanism [13]. There were some TDs penetrated into 

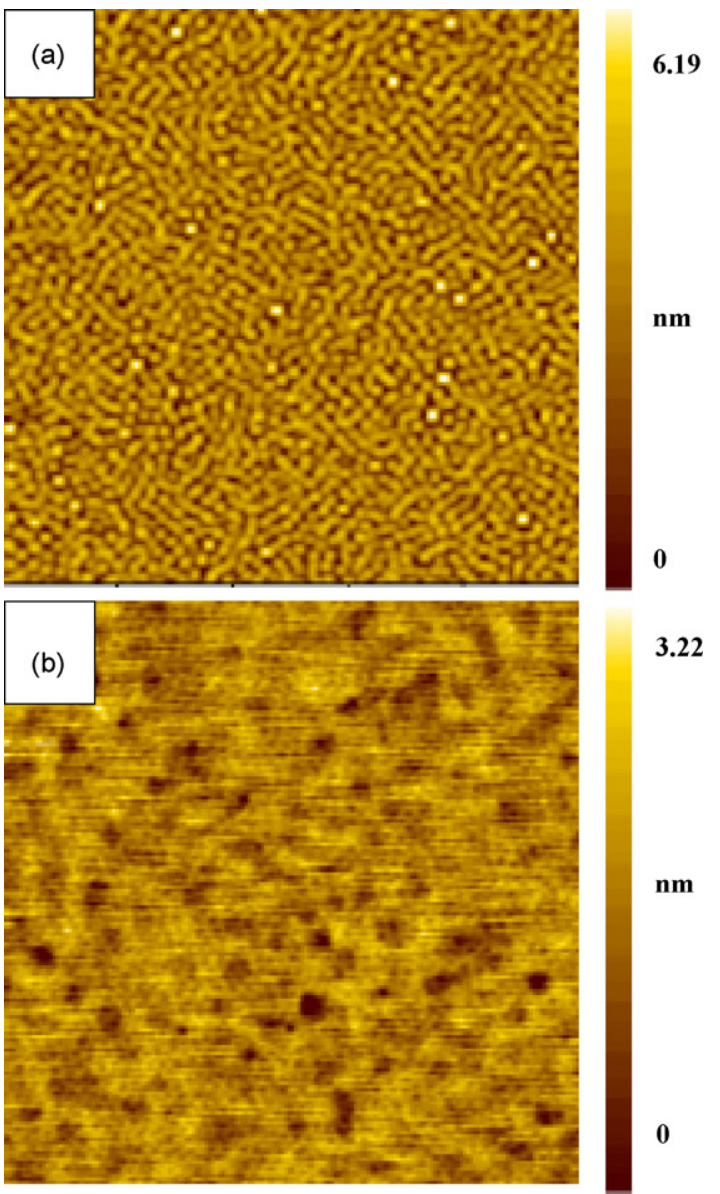

3.22

nm

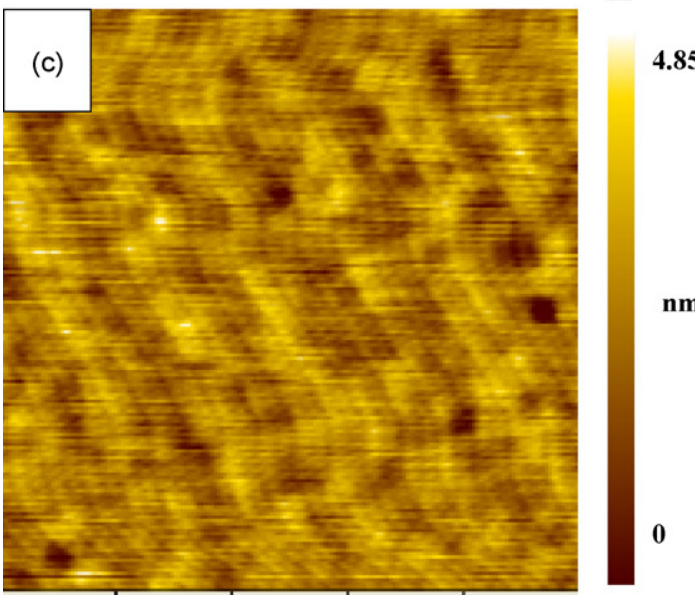

Fig. 3. AFM scanned image $(2 \mu \mathrm{m} \times 2 \mu \mathrm{m})$ showing the surface morphology of (a) Ge QDs capped with a 20-nm-thick Si cap layer, (b) m-SiGe islands with a 20-nmthick Si cap layer, and (c) 10-period stacked m-SiGe/Si bilayers.

$\mathrm{Si}_{0.8} \mathrm{Ge}_{0.2}$ film on $\mathrm{Si}$ form the observation of XTEM. Fig. 4(b) shows an XTEM image of the 500 -nm-thick $\mathrm{Si}_{0.8} \mathrm{Ge}_{0.2}$ film grown on the 10-period of $\mathrm{m}$-SiGe QDs stacked layer. It is evident that the misfit dislocations are mainly confined in the buffer layer including $\mathrm{m}$ SiGe islands, leaving the SiGe overlayer with a low TD density. This result indicates that the multilayer buffer of m-SiGe islands can provide enough effective nucleation sites for the occurrence of misfit dislocations (MDs), this buffer is also very effective in increasing the length of MDs and blocking the propagation of TDs. Moreover, the characteristic feature of MFR mechanism, i.e. pile-up of dislocations to several microns deep into Si substrate, is absent
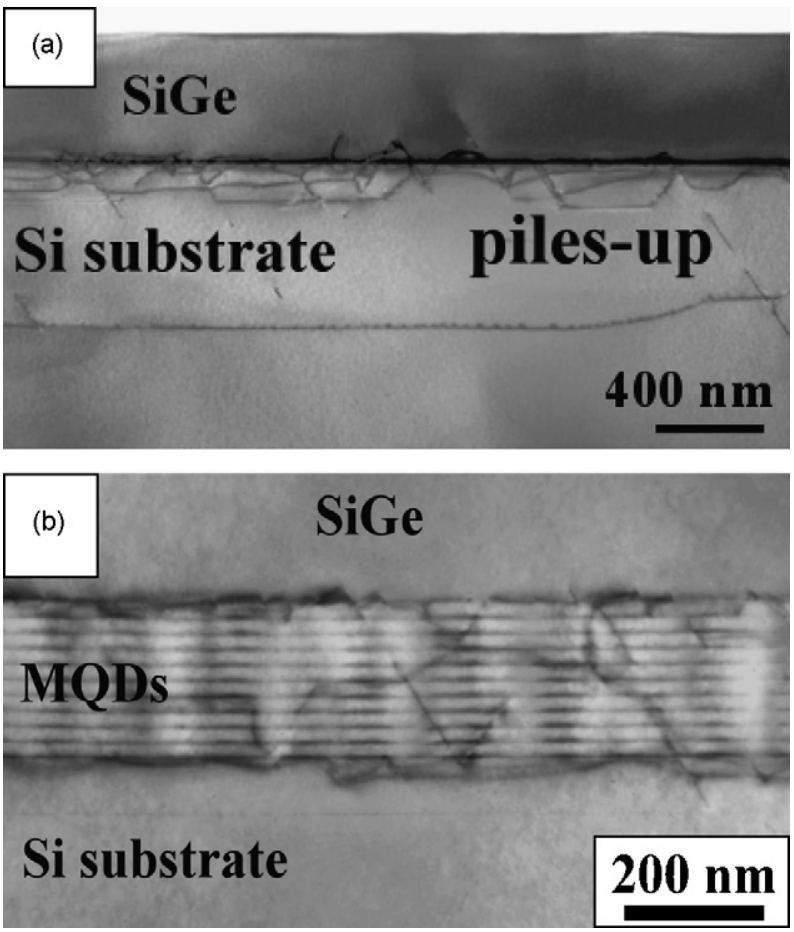

Fig. 4. XTEM images of (a) $\mathrm{Si}_{0.8} \mathrm{Ge}_{0.2}$ film grown on the 10 -period $\mathrm{m}$-SiGe islands/Si bilayers and (b) magnified view of a selected region of the buffer layer.

in the XTEM image (Fig. 4(b)). The stacks of m-SiGe islands with Si spacers apparently improve the homogeneity and periodicity of islands and create a uniform strain undulation on the surface, which is expected to relax the strain during the subsequent SiGe growth. This mechanism is rather similar to the case of Sakai et al. [16]. The dominant relaxation mechanism in SiGe may be introduced by the generation of MDs from the top layer of $\mathrm{m}$ SiGe islands rather than the multiplication of pre-existing MDs via MFR mechanism. The estimated results of TD densities were listed in Table 1. The TD density of sample D is about four orders of magnitude lower than that obtained from the samples without using any $\mathrm{m}$-SiGe islands layers, and compares favorably with that grown with a CGBs layer as buffer $\left(5.1 \times 10^{5} \mathrm{~cm}^{-2}\right)$.

According to the AFM image of relaxed SiGe epilayer, the different amplitude of dislocations pile-up show the number of multiplications, and the different spatial periodicity reflects the nucleation centers of MDs. The AFM images of as-grown samples B and D are shown in Fig. 5(a) and (b). The RMS of roughness was measured to be about 5.2 and $3.1 \mathrm{~nm}$, respectively. This value are both smaller than that of the control sample with a CGB layer $(\sim 6.4 \mathrm{~nm})$ and $\mathrm{Si}_{0.8} \mathrm{Ge}_{0.2}$ uniform layer $(\sim 12 \mathrm{~nm})$, in which the strain is expected to be relaxed by the MFR mechanism, which lead to a larger undulation of the cross hatch due to the pile-up of buried dislocations. Using the $\mathrm{m}-\mathrm{SiGe}$ islands as buffer, the relaxation mechanism of SiGe uniform has been changed based on the observation of AFM. The spatial periodicity of sample B is higher than that of sample $D$, this result suggests that 1-period of m-SiGe as buffer can provide more nucleation centers for the generation of MDs than that of 10-period. The density of Ge islands for a single layer is $\sim 10^{10} \mathrm{~cm}^{-2}$. However, for the sample of 10 -period stacked layer, the density of Ge dots reduced only $5 \times 10^{9} \mathrm{~cm}^{-2}$. Hence, in the single $\mathrm{m}$-SiGe layer may own more nuclei for the occurrence of MDs during the growth of SiGe overlayer. Form the AFM image of sample $B$, in which the buffer layer contains a single $\mathrm{m}$-SiGe island layer, the sample was found to have a lot of small pits, that possibly 

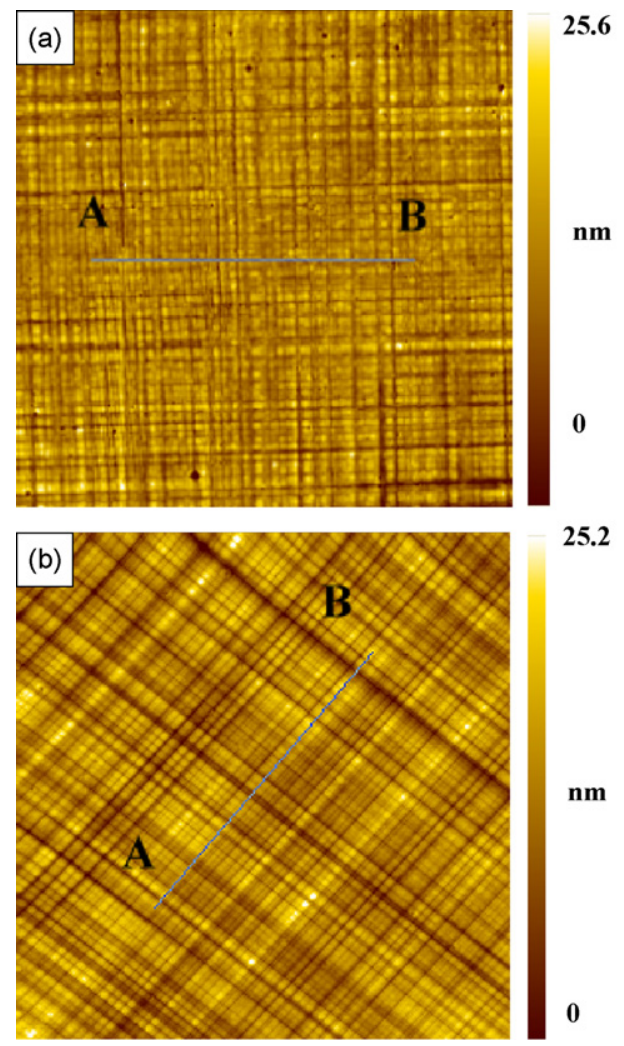
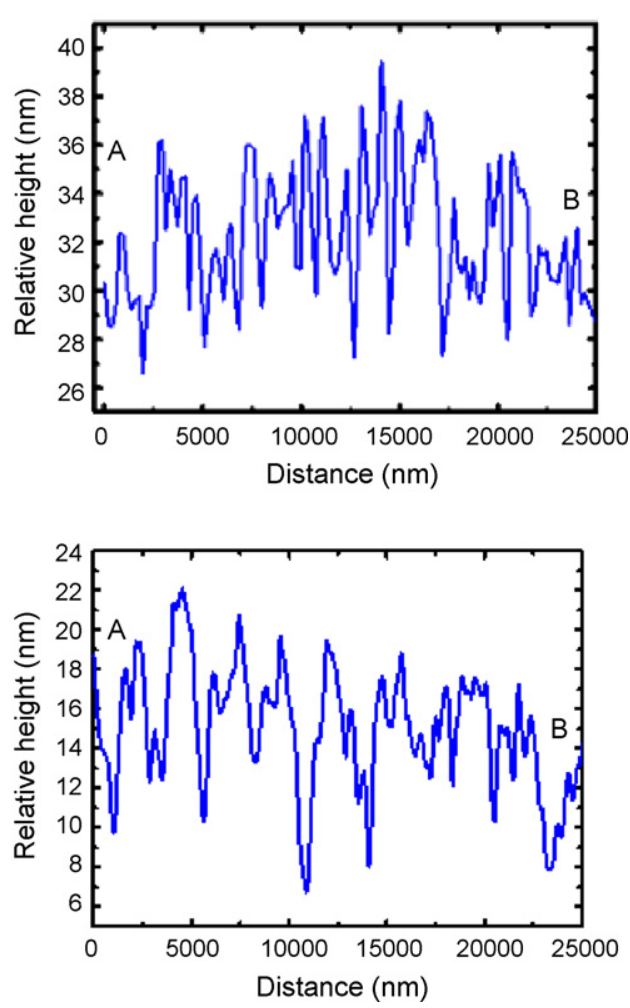

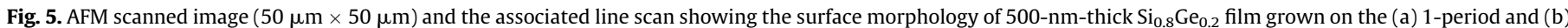
10 -period of $\mathrm{m}$-SiGe islands/Si bilayers.

originated form the TDs in the uniform SiGe layer. Fold number of $\mathrm{m}$-SiGe can enlarge the length of MDs, further avoid the generation of TDs as well as act as the filter of TDs. The relaxations, surface roughness of the relaxed SiGe, are functions of period number in $\mathrm{m}$-SiGe multilayers and listed in Table 1 . Surface roughness of the relaxed $\mathrm{SiGe}$ reaches a minimum as 10 -period $\mathrm{m}-\mathrm{SiGe} / \mathrm{Si}$ as buffer. However, relaxation in SiGe overlayer peaked when the period number in multilayer m-SiGe layer is 10. The FWHM of XRD spectrum from the SiGe is also a function of periods of $\mathrm{m}-\mathrm{SiGe}$ islands. These experiment results suggest that 10-period of $\mathrm{m}-\mathrm{SiGe}$ islands stacked layer serves as an excellent template for the 500nm-thick relaxed SiGe with a smooth surface, a low TD density, and a required relaxation.

\section{Conclusions}

A useful approach to grow the high-quality relaxed SiGe epilayers with a buffer layer containing modified SiGe islands and required relaxation is developed. The pre-intermixing procedure of $\mathrm{m}$-SiGe layer with flat surface is composed of 28 eq-MLs $\mathrm{Si}$ encapsulation layer on 13.1 eq-MLs Ge islands and a subsequent in situ annealing of $10 \mathrm{~min}$. The $\mathrm{m}$-SiGe with non-uniformity of $\mathrm{Ge}$ content can provide centers for nucleation sites for the generation of MDs for the following uniform SiGe layer, increase the length of MDs, and retard the propagation of TDs. With multiple m-SiGe QDs layers as a buffer, the resulting relaxed SiGe films possess excellent structural integrity compared with those formed by the MFR mechanism. The $\mathrm{Si}_{0.8} \mathrm{Ge}_{0.2}$ films of $500 \mathrm{~nm}$ grown on the 10 -period $\mathrm{m}$-SiGe/Si bilayers have a TD density of $2.0 \times 10^{5} \mathrm{~cm}^{-2}$ and a strain relaxation of $89 \%$.

\section{References}

[1] J.-M. Baribeau, N.L. Rowell, D.J. Lockwood, J. Mater. Res. 20 (2005) 3278.

[2] J. Wan, Y.H. Luo, Z.M. Jiang, G. Jin, J.L. Liu, K.L. Wang, X.Z. Liao, J. Zou, Appl. Phys. Lett. 79 (2001) 1980.

[3] Y.H. Peng, C.-H. Hsu, P.S. Chen, M.-J. Tsai, C.H. Kuan, C.W. Liu, Appl. Phys. Lett. 85 (2004) 6107

[4] A. Rastelli, M. Kummer, H. von Kanel, Phys. Rev. Lett. 87 (2001) 256101.

[5] P. Sutter, M.G. Lagally, Phys. Rev. Lett. 81 (1998) 3471.

[6] Z. Zhong, J. Stangl, F. Schäffler, G. Bauer, Appl. Phys. Lett. 83 (2003) 3695.

[7] D. Dental, J.L. Bischoff, L. Kubler, J. Werckmann, M. Romeo, J. Cryst. Growth 191 (1998) 697.

[8] J. Huang, Z. Ye, W. Chen, Z. Qi, H. Lu, W. Lei, B. Zhao, D. Que, J. Cryst. Growth 206 (1999) 294.

[9] M.L. Lee, E.A. Fitzgerald, M.T. Bulsara, M.T. Currie, A. Lochtefeld, J. Appl. Phys. 97 (2005) 011101.

[10] Y.H. Luo, J. Wan, R.L. Forrest, J.L. Liu, G. Jin, M.S. Goorsky, K.L. Wang, Appl. Phys. Lett. 78 (2001) 454.

[11] H. Trinkaus, B. Holländer, St. Rongen, S. Mantl, H.-J. Herzog, J. Kuchenbecker, T. Hackbarth, Appl. Phys. Lett. 74 (2000) 3552.

[12] A.R. Powell, S.S. Iyer, F.K. LeGoues, Appl. Phys. Lett. 64 (1994) 1856

[13] S.W. Lee, P.S. Chen, T.Y. Chien, L.J. Chen, C.T. Chia, C.W. Liu, Thin Solid Films 508 (2006) 123.

[14] P.S. Chen, S.W. Lee, Y.H. Peng, C.W. Liu, M.-J. Tsai, Phys. Status Solid 241 (2004) 3650 .

[15] D. Schmmel, J. Electrochem. Soc. 126 (1979) 479

[16] A. Sakai, K. Sugimoto, T. Yamamoto, M. Okada, H. Ikeda, Y. Yasuda, S. Zaima, Appl. Phys. Lett. 79 (2001) 3398. 